\title{
Auf dem Weg zu Nicht-Flektierbaren: Die Deflexion der deutschen Eigennamen diachron und synchron
}

\author{
Damaris Nübling
}

\section{Einleitung}

Im heutigen Deutsch sorgt die Flexion von Eigennamen im Genitiv für einen echten Zweifelsfall, mehr noch bei geographischen Namen als bei Personennamen, vgl. des Orinoko(s), des $\operatorname{Iran}(s)$, des vereinigten Deutschland(s), ebenso im Plural: die beiden Deutschland(s). Personennamen werden, wenn ihnen ein Artikel (mit oder ohne Adjektiv) vorangeht, in aller Regel schon nicht mehr flektiert, vgl. die 1. Auflage (1774) von „Die Leiden des jungen Werthers" mit Genitivendung mit der 2. Auflage (1787), wo diese Endung schon fehlt. Heute dominiert die Nichtflexion: der Geburtstag des kleinen Julian, des Helmut Kobl. Aus diachroner Sicht stellt dieses Stadium nur einen weiteren Schritt in Richtung onymische Deflexion dar. Dieser Deflexion und ihren Gründen soll in diesem Beitrag nachgegangen werden. Flektierten Eigennamen im Althochdeutschen noch ausgiebig (in mehreren Flexionsklassen), so haben sie im Laufe der Zeit ihre Flexion in zweierlei Hinsicht stark eingeschränkt: a) paradigmatisch durch den Abbau an Allomorphie und die Durchsetzung sog. überstabiler Marker, die oft erstes Indiz für den Beginn von Deflexion sind; b) syntagmatisch durch den sukzessiven Abbau von Flexiven am Wortkörper. Neben Kasus und Numerus haben sich auch bei Genus tiefgreifende Veränderungen vollzogen: Genus wird zunehmend pragmatisch „,von außen“ fixiert, d.h. immer mehr von Eigenschaften des Referenzobjekts gesteuert.

\section{Die Eigennamen(de)flexion diachron}

Die onymische Deflexion beginnt etwa ab mhd. Zeit. Im Ahd. entspricht die Flexion der Eigennamen weitestgehend der der Appellative, in einigen Deklinationsklassen übertrifft sie diese sogar. Im Folgenden gehen wir die drei Nominalkategorien einzeln durch, um anschließend zu einem Überblick zu gelangen. Wir beginnen mit Genus, um dann zu Kasus und Numerus überzugehen. 


\section{$2.1 \quad$ Genus}

Es ist bisher nicht viel zum Komplex Eigenname und Genus gearbeitet worden. Doch lässt sich soviel sagen, dass die Eigennamen im Laufe der Zeit immer mehr von einem lexikalischen (grammatischen) zu einem semantischen bzw. vielmehr zu einem referentiellen (pragmatischen) Genus übergehen, d.h. von einem rein inhärenten, arbiträren Genus zu einem weniger arbiträren, dessen Zuordnung sich aus bestimmten Eigenschaften des Denotats bzw. des konkreten Referenten (Objekt) ergibt. Köpcke/ Zubin $(1983,1984,1996,2005,2009)$ haben sich mehrfach mit dieser Frage befasst und stellen fest, dass manche Bereiche der Appellativik nur lexikalisches Genus enthalten, z.B. die Gabel, der Löffel, das Messer, andere - die Mehrheit - jedoch einer Systematik folgen wie etwa die Bezeichnungen für Früchte, die feminin klassifiziert werden (die Birne, Kiwi, Mango, Ananas) ${ }^{1}$ oder Bezeichnungen für Alkoholika, die maskulin klassifiziert werden (der Wein, Whisky, Baileys). Ein solches semantisches Genus gilt auch für die Bezeichnungen höherer Lebewesen (Menschen, Säugetiere), wo Sexus Genus steuert. Nach Köpcke/Zubin folgt die Genuszuweisung fast immer bestimmten lexikalisch-semantischen Feldern, d.h. der Arbitraritätsfaktor ist geringer als gemeinhin vermutet.

Das semantische Genusprinzip gilt seit jeher für die Personennamen: Diese folgten schon bei den dithematischen Rufnamen im Germanischen und im Althochdeutschen einer strikten Sexus-Genus-Relation, die für das Zweitglied, also das Kopfnomen galt: die ahd. Feminina -bilt (zu biltia ,Kampf'), -borg/-burg ,Schutz', -beid,Art, Wesen' waren als Zweitglieder nur in Frauennamen möglich, die Maskulina -frid (zu fridu, Friede), -braban ,Rabe' oder -gèr ,Speer dagegen nur in Männernamen. Neutra kamen hier also nicht vor. Das Genus des Erstglieds war dagegen irrelevant, hier kamen alle drei Genera vor unabhängig vom biologischen Geschlecht des Denotats

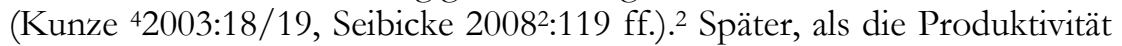
dieses kombinatorischen Namenbauprinzips erlosch und mehr monothematische, aber auch kontrahierte Namen aufkamen und dieses System verunklarten, traten oft spezifische Endungen an: $-a$ und $-e$ für Frauen, $-o$ für Jungen: Adel-a (zu Adelheid), doch auch Vollformen wie Kriembild-e, Kunigund-e konnten erweitert werden, vs. Ott-o (zu Otfrid), Benn-o (zu Bernhard). ${ }^{3}$

1 Die beiden einzigen Ausnahmen sind die (älteren) Bezeichnungen Apfel und Pfirsich. Die Maskulinklassifikation ist nicht mehr produktiv.

2 Auch Adjektive konnten an der Rufnamenbildung teilhaben, sowohl als Erst- wie auch als Zweitglied.

3 Diese vokalische „Auffrischung“ zwecks klarer Sexuskodierung (Berte $>$ Berta, Otte $>$ Otto) hebt Steche (1927) wie folgt hervor: „Diese Wiederbelebung klangvoller Endsilben ist ein ganz außergewöhnlicher Vorgang in der deutschen Sprachgeschichte“ (141). 
Damit entwickelte sich die Genus/Sexus-Angabe zu einem overt-formalen Prinzip, während die Genushaltigkeit des Zweitglieds als kovert-formal zu gelten hat. Diese vokalisch auslautenden (Kurz-)Formen wurden schwach flektiert, die älteren dithematischen, konsonantisch auslautenden Namen dagegen stark (s.u. in Abschnitt 2.1).

Da die germanischen Rufnamen heute, sofern überhaupt gebräuchlich, kaum noch segmentierbar (vgl. kontrahiertes Rolf $<$ Rudolf $<*$ broP,Ruhm ${ }^{6}+$-wolf, Wolf') und, falls doch, bzgl. ihres Zweitglieds kaum noch einem Genus zuzuordnen sind (wie noch etwa bei Siegfried), ist dieses Letztglied-Genusprinzip obsolet geworden. Sexus wird heute entweder overt-formal über bestimmte Endungen markiert (s. die oben genannten Vokale, aber auch Suffixe wie -ine, vgl. Bernhardine) oder, was der häufigere Fall ist, konventionell realisiert, d.h. es wird „gewusst" wie bei Doris (+) vs. Boris ( $\left.{ }^{\top}\right)$. Immer häufiger wird es subtil am gesamten Wortkörper markiert, etwa indem Männernamen durchschnittlich mehr Konsonanten enthalten bzw. auf solche auslauten, weniger Silben umfassen und eher auf der ersten Silbe betont werden als Frauennamen, die im Schnitt mehr Vokale und mehr Silben enthalten und seltener Erstsilbenakzent aufweisen (s. hierzu ausführlich Oelkers 2003, auch Nübling 2009)..$^{4}$ Wichtig ist bei alledem: Hier handelt es sich primär um Indikatoren für Sexus - dem dann die Genuszuweisung an den Substantivbegleitern (Artikel, Adjektiv) folgt. Im Gegensatz zu früher ist jedoch kein Bestandteil des Namens selbst mehr genushaltig. Sexus als semantische Information des Namens hat noch mehr an Stärke gewonnen. Damit haben Rufnamen heute einen höheren Gehalt an semantischem Genus als früher. ${ }^{5}$ Diese Prinzipien können nur gebrochen werden, wenn Namen diminuiert werden: Dann nehmen sie nach dem Kopf rechts-Prinzip neutrales Genus an (das Hänschen, das Mariechen). ${ }^{6}$

Auch Familiennamen folgen strikt dem Sexus-Genus-Prinzip, enthalten jedoch selbst keinerlei Informationen über diese Kategorien, nicht einmal konventionell: Hier gilt ausschließlich das referentielle (oder pragmatische) Genuszuweisungsprinzip, d.h. erst die Kenntnis des biologischen Geschlechts des konkreten Referenzobjekts weist Sexus und damit Genus

4 Im Oberdeutschen werden Personennamen regelmäßig von einem Definitartikel begleitet, der die Sexus- und damit Genusmarkierung leistet.

5 Sexus ist das einzige Sem, das der - ansonsten bedeutungslose - Rufname enthält. Dies rechtfertigt die Rede vom semantischen Genus. Dass heute manchmal „falsche Zuordnungen" realisiert werden (Nicola oder Luca für Jungen, Robin für Mädchen), belegt die Spannung zwischen semantischem und referenziellem Genus und damit die Existenz eines semantischen, onymischen Genus.

6 Diese morphologische Genuszuweisung scheint aber, sobald der Name selbständig wird, d.h. nicht mehr nur als Ableitung fungiert, von Sexus überlagert werden zu können: So sind die Diminutiva Christel und Bärbel Feminina und keine Neutra. 
zu. Kein deutscher Familienname, auch nicht einer auf -mann, enthält Indikatoren für Genus oder Sexus, es kommt hier allein auf das konkrete Referenzobjekt an: die (Frau) Baumann/Meier - der (Herr) Baumann/Meier. In anderen Sprachen wie dem Polnischen, Russischen, Lettischen und Litauischen gibt es dagegen durchaus sexus- und damit genushaltige Familiennamensuffixe. ${ }^{7}$

Was andere Namenklassen betrifft, so mangelt es hier an diachroner Forschung. Fest steht, dass auch hier eine Zunahme an semantischem bzw. pragmatisch-referentiellem Genus stattgefunden hat, denn heute gilt z.B., dass Städtenamen immer Neutra sind. Hier waren im Ahd. noch alle drei Genera möglich. Im Frühnhd. wurden Städte durchgehend feminin klassifiziert, während sie erst im 18./19. Jh. zu Neutra wurden, vgl. die Rom $>$ das Rom (des Mittelalters), die $>$ das Zürich, die $>$ das Athen etc. Das früher noch gültige Kopfprinzip (Paul 1917:\$117) ist obsolet geworden, vgl. der Berg, aber das (alte) Heidelberg, die Burg, aber das Freiburg, die Stadt, aber das Neustadt. Gründe für diesen Genuswechsel sind bislang nicht bekannt. Fleischer (1970) sieht in den Genusunterschieden eine Homonymenvermeidung zwischen Namen und Appellativen und den Namen verschiedener Namenklassen.

Im Deutschen gilt, dass Wüsten immer Feminina sind (die Sahara, die Namib), ebenso Schiffs-, Motorrad- und Flugzeugnamen (die Kaiser Wilhelm der Große, die Titanic - die BMW - die Landshut), während Autonamen Maskulina sind (der Golf, der Mercedes, der Tiguan; hierzu s. Köpcke/Zubin 2005, die solche Autonamen als kopflose NPs analysieren). Hotel- und Restaurantnamen sind Neutra (das Steigenberger, das Ibis), ebenso Ländernamen (sofern sie keinen festen Artikel mit sich führen). Man kann die Wirksamkeit dieses Prinzips an ein und demselben Namen durchspielen, vgl. die Continental (Schiff, Motorrad, Flugzeug) vs. der Continental (Auto) vs. das Continental (Hotel, Restaurant; zu solchen onymischen Mehrfachnutzungen gleicher Wörter s. Köpcke/Zubin 2005:113/114). Diese Zuweisungen waren historisch nicht so fest. Es gilt bei der Genusfixierung auch nicht das Prinzip, dass sich das Genus der entsprechenden appellativischen Klassenbezeichnung durchsetzt (das bei Stadt ja feminin und bei Schiff und Auto neutral wäre). Die Genuszuweisung erfolgt somit pragmatisch und nach im Einzelnen nach noch nicht bekannten Prinzipien (warum sind Autonamen maskulin und Städtenamen neutral?). ${ }^{8} \mathrm{Um}$ ihr Genus sichtbar zu machen, führen die meisten dieser Namen den Artikel fest mit sich.

7 Früher (und teilweise noch heute) gab es dies auch in deutschen Varietäten, vgl. die Müllersche, die Müllerin/Müllern für die Ehefrau eines Herrn Müller.

8 (Noch) nicht fest ist das Genus bei Berg- und v.a. bei Gebirgs- und Flussnamen: der Har: die Eifel - der Neckar, die Donau. Sind sie in ihrer Bildung transparent, so gilt hier wie in der Appellativik das Grundwortprinzip: das Matterhorn, die Zugspitze, der Feldberg. 
Damit leistet das deutsche Genus bei den meisten Eigennamen eine echte Nominalklassifikation, wie man dies eher aus Sprachen wie dem Suahili oder Chinesischen kennt.

Aufschlussreich für die Genusfixierung bei Eigennamen könnten Namen von GmbHs und Aktiengesellschaften sein, die wegen ihres syntaktischen Kopfs Gesellschaft Feminina sind. Hier kommt es immer häufiger zu elliptischen (doch weiterhin durchweg femininen) Bildungen wie (die) deutschepapier, Deutsche Beteiligungs, Deutsche Euroshop, d.h. diese Wortkürzungen übernehmen das Genus ihres einstigen sog. Sockels oder syntaktischen Kopfs (des Letztglieds $A G$ ) und generalisieren es womöglich langfristig (hierzu s. Fahlbusch 2009). Genau hier kann man den Verlust syntaktischer Köpfe beobachten. Ähnliches schreibt Steche (1927:81) in Bezug auf das durchgehend neutrale Genus bei Städte- und Ländernamen (das alte Rom, das südliche Spanien), wo er auch den Verlust des Kopf rechtsPrinzips anspricht:

„Aber diese Einheitlichkeit ist geschichtlich sehr jung; im Ahd. und Mhd. konnten die Namen der Orte und Länder jedes Sprachgeschlecht haben. Heute sind die Ortsnamen und deutewortlosen Ländernamen dadurch scharf von den Gattungswörtern geschieden, dass bei ihnen die erste und sechste Geschlechtsregel, wonach Zusammensetzungen dasselbe Geschlecht wie das Grundwort haben und Wörter auf - $e$ weiblich sein müssen, nicht gelten“.

Die Formulierung ,jedes Sprachgeschlecht“ deutet zumindest auf die frühere Gültigkeit des Kopf rechts-Prinzips hin.

Etwas anders argumentiert die Duden-Grammatik (2009:\$251), die das Genus der entsprechenden Gattungsbezeichnung auf die Namen vererbt sieht, wenn sie angesichts zwischen Femininum und Neutrum schwankender sog. Firmennamen wie Nestlé und Apple die feminine Genuszuweisung wie folgt begründet:

„Das feminine Geschlecht kann man damit erklären, dass man sich eine Gattungsbezeichnung wie Firma, Gesellschaft oder Gruppe hinzudenken kann; die genannten femininen Substantive kommen zugleich als Oberbegriffe infrage. Man kann das Genus zwar nicht am Firmennamen selbst ablesen, wohl aber an wiederaufnehmenden Wörtern, zum Beispiel Possessiven [es folgen Internet-Belege, $\mathrm{DN}]$ : Nestlé hat seinen Halbjahresgewinn dramatisch erhöht [...]; Nestlé hat $\underline{\text { ih- }}$ ren Umsatz [....] gesteigert [...]“.

Köpcke/Zubin (2005) argumentieren jedoch im Fall der maskulinen Autonamen dagegen, dass sie das Genus von elliptischem Wagen erben, sondern dafür, dass es sich hier um feldexterne (außersprachlich motivierte) Effekte handelt. Was jedoch bei dieser Arbeit zur pragmatischen Genuszuweisung bei Eigennamen fehlt, ist eine Begründung für die konkrete Auswahl des jeweiligen Genus und, mehr noch, eine Begründung dafür, dass dieses Genus historisch wechseln kann, wie dies für die Städtenamen belegt ist (Femininum $>$ Neutrum). 
Fazit: Während die appellativische Genuszuweisung primär lexikalischen oder semantischen Zuweisungsprinzipien folgt, tendiert die onymische Genuszuweisung im Fall der Rufnamen zum semantischen ${ }^{9}$, ansonsten, d.h. im Fall der Familiennamen, der Topo- und der Ergonyme (Städte, Schiffe, Länder, Autos etc.), stark zum pragmatischen Genus. Diese Divergenz vergrößert sich diachron (s. hierzu auch Thieroff 2000, der im Fall von Kosovo feststellt, dass es im Zuge der Entwicklung von einem Gebiets- zu einem Ländernamen zunehmend neutral klassifiziert wird und dabei auch seinen Artikel ablegt: der/das Kosovo > Kosovo (n.)).

\section{$2.2 \quad$ Kasus}

\subsubsection{Die Flexion im Alt- und im Mittelhochdeutschen}

Die onymische Kasusflexion entspricht im Ahd. noch weitestgehend der appellativischen: Die Eigennamen partizipieren in gleicher Weise an den verschiedenen Deklinationsklassen. Dabei gilt grob folgende Regel: Konsonantisch auslautende Namen flektieren stark (wobei das Letztglied die Klasse steuert), vokalisch auslautende schwach. Da Plurale gemäß Paul (1917:158) ursprünglich nicht gebildet wurden, beschränken wir uns auf die Singularflexion, zunächst auf die der starken Klassen (s. Tabelle 1). Hierin befanden sich zusammengesetzte Rufnamen wie Hludowig, Werinbrabt, aber auch Fremdnamen auf Konsonant wurden integriert, s. Petrus (ebenso: Adam, David etc.). Bei den dithematischen Rufnamen scheint es üblich gewesen zu sein, die Nom.Sg.-Endung zu eliminieren, z.B. Namen auf -frid < fridu, -bilt < biltia, -rün < rüna, Geheimnis'.

\begin{tabular}{|c|c|c|c|c|c|}
\hline Kasus & $\begin{array}{l}\text { Mask. stark } \\
\text { (im Sg. } \\
a=i-\mathrm{Kl} \text {.) }\end{array}$ & $\begin{array}{l}\text { Mask. } \\
\text { stark } \\
\text { (Fremd- } \\
\text { name) }\end{array}$ & $\begin{array}{l}\text { Fem. stark } \\
(\text { (j)o-St.) }\end{array}$ & $\begin{array}{l}\text { Fem. } \\
\text { stark } \\
(i-\mathrm{Kl} .)\end{array}$ & $\begin{array}{l}\text { Mask. stark } \\
u \text {-K1.: } \\
\text { OrtsN }\end{array}$ \\
\hline $\begin{array}{l}\text { Nom. } \\
\text { Gen. } \\
\text { Dat. } \\
\text { Akk. }\end{array}$ & $\begin{array}{l}\text { Hartmuot } \\
\text { Hartmuot-es } \\
\text { Hartmuot-e } \\
\text { Hartmuot-an }\end{array}$ & $\begin{array}{l}\text { Petrus } \\
\text { Petrus-es } \\
\text { Petrus-e } \\
\text { Petrus-an }\end{array}$ & $\begin{array}{l}\text { Gundrūn-Ø } \\
\text { Gundrūn-a } \\
\text { Gundrūn-u } \\
\text { Gundrūn-a }\end{array}$ & $\begin{array}{l}\text { Hiltigart } \\
\text { Hiltigart- } \overline{1} \\
\text { Hiltigart- } \overline{1} \\
\text { Hiltigart }\end{array}$ & $\begin{array}{l}\text { Stetifurt-u } \\
\text { Stetifurt-es } \\
\text { Stetifurt-iu } \\
\text { Stetifurt-u }\end{array}$ \\
\hline
\end{tabular}

Tab. 1: Die starke Flexion bei Eigennamen im Ahd.

9 Allerdings können Rufnamen auch für andere Objektgruppen vergeben werden. Dann weicht die semantische Genuszuweisung der pragmatisch-referentiellen: das Doris/das Boris wäre z.B. ein Hotel oder ein Restaurant. Auch der weibliche spanische Rufname Mercedes ist als Autoname maskulin klassifiziert: der Mercedes. 
Als Besonderheit bei den Maskulina ist auf die Akkusativendung -an hinzuweisen: Hartmuotan, Jesusan, Adaman, Hluduigan, Petrusan etc. (Schatz 1927:252). Sie kam bei genuinen Appellativa nicht vor. ${ }^{10}$ Sie entstammt der Adjektivflexion, wahrscheinlich vermittelt durch adjektivische Zweitglieder, wie sie bei dithematischen Rufnamen häufig vorkamen, vgl. Namen auf ahd. -bald ,kühn', -berabt, glänzend', -liob ,lieb', z.B. Uuerinbrabtan. Dadurch wurde eine Opposition zum ansonsten synkretistischen Nominativ geschaffen und eine klare Agens-/Patiens-Unterscheidung gewonnen. Personennamen bezeichnen immer belebte und damit agensfähige Denotate. Auch bei den Feminina ist eine Nominativ-/Akkusativ-Opposition eingetreten, indem hier der Nominativ im Gegensatz zu den Appellativen (die hier $-a$ enthalten) eine Nullendung trägt, die möglicherweise den Maskulina entstammt. Damit praktizieren die Personennamen mehr Kasusdifferenzierungen als die Appellative.

Tabelle 2 weist die schwache Flexion bei den Eigennamen aus. Hierzu gehörten die vokalisch auslautenden Namen, die oft auf Kurz- oder Kontraktionsformen aus vollen Namen zurückgehen, oder die in vokalisch auslautenden Fremdnamen (wie Maria oder Georio), oft auch in einer Erweiterung voller, dithematischer Namen (Kriembilde). Wie man sieht, kommt es hier nur zu einer morphologischen ,+/- Nominativ'-Opposition.

\begin{tabular}{|l|ll|ll|}
\hline \multicolumn{1}{|c|}{ Kasus } & \multicolumn{2}{|c|}{ Feminina } & \multicolumn{2}{c|}{ Maskulina } \\
\hline Nom. & Uot-a & Mari-a & Ott-o & Geori-o \\
Gen. & Uot-ūn & Mari-ūn & Ott-en/-in & Geori-en/-in \\
Dat. & Uot-ūn & Mari-ūn & Ott-en/-in & Geori-en/-in \\
Akk. & Uot-ūn & Mari-ūn & Ott-on/-un & Geori-on/-un \\
\hline
\end{tabular}

Tab. 2: Die schwache Flexion bei Eigennamen im Ahd.

10 Doch wurde die Endung durchaus auf Appellativa übertragen, die wie Eigennamen gebraucht wurden: ahd. fateran, trubtinan (Schatz 1927:252). 


\subsubsection{Paradigmatischer Flexionsabbau}

Im weiteren Verlauf der Sprachgeschichte finden nach Paul (1917:153 ff.) Ausgleiche zwischen starker und schwacher sowie zwischen femininer und maskuliner Flexion statt.

Durch die Endsilbenabschwächung im Mittelhochdeutschen fallen die starken und schwachen maskulinen Akkusative zu -en zusammen. Diese flexivische Überschneidung führte dazu, dass im Dativ immer öfter schwaches -en auf starke Rufnamen übertragen wurde, seltener auch starkes $-e$ auf schwache (männliche) Namen (Paul 1917:\$108; Paul 2007:199):

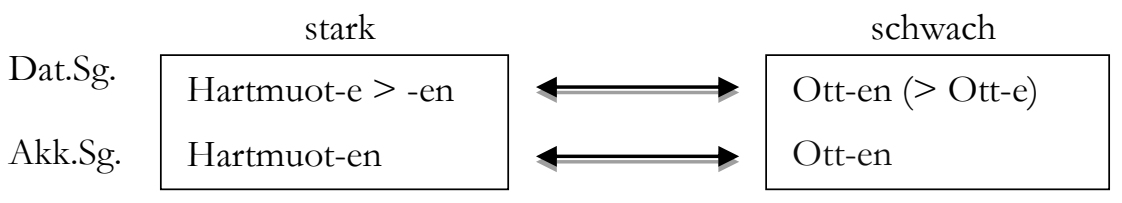

Später fanden von hier aus auch Übertragungen auf Familiennamen statt (ich begegnete Scbillern; Klopstocken scbien das Bild zu niedrig; Blatz 1900:336), auBerdem auf onymisch verwendete Appellativa, vgl. ich bin bei / ich gehe zu Muttern, Vatern. Damit entwickelt sich die Endung -en zum über- oder superstabilen (d.h. flexionsklassenübergreifenden) Marker für Dativ und Akkusativ. Bei den Feminina breitet sich auch die schwache auf Kosten der starken Flexion aus: Immer mehr starke Namen nehmen im Nom.Sg. ein $-e$ an (Kunigunde, Kriembilde) sowie $n$-haltige Dative und Akkusative $(K u$ nigunden, Kriembilden; s. Paul 1917:\$109). Aber auch solche ohne -e im Nom. flektieren schwach: Nom. Gertrud - Dat./Akk. Gertruden (Blatz 1900:338).

Auch der Genitiv entwickelt mit der Ausbreitung der starken, maskulinen $s$-Endung einen überstabilen Marker, der sich zunächst suffigierend mit der schwachen en-Endung verbindet: Reinek-en-s, Carl-en-s, Franz-en-s. Diese gestärkte Doppelendung -ens hat sich übrigens bis heute bei Genitiven solcher (einsilbiger) Namen erhalten, die auf [s] auslauten: Hans-ens, Marx-ens. Ansonsten ersetzt der $s$-Genitiv die alte en-Endung bei den schwachen Maskulina (Ott-en > Otto-s), um dann sogar auf die Feminina überzugehen: Sofern weibliche Rufnamen im Nom. nicht auf $-e$ auslauten (hier hält sich der alte $e n$-Genitiv länger), erhalten auch sie den einstmals starken $s$-Genitiv, nicht selten auch die doppelte ens-Endung: Kriembild-ens / Kriembild-s, Gertrud-ens / Gertrud-s, Cleopatr-ens / Cleopatra-s, Anna-s, Maria$s$. Heute bekommen sämtliche Feminina den $s$-Genitiv, auch solche auf $-e$ : Susanne-s, Marianne-s. 
Was den $s$-Genitiv betrifft, so ist hier eine weitere Besonderheit interessant: Bei den Eigennamen wird die ansonsten geltende, phonologisch konditionierte Allomorphie zwischen der langen es- und der kurzen $s$-Endung, die sich nach dem Auslaut, dem Umfang und der Prosodie des betreffenden Appellativs richtet, unterdrückt, vgl. die appellativische Alternanz bei des Tages - des Feiertags, des Kindes - des Enkelkinds (Szczepaniak 2010). Dagegen gilt bei Eigennamen ausschließlich uniformes -s: des Kindes, aber Patrick Süskinds (*Süßkindes) / Wilhelm Buschs / Max Frischs Werk. Dieses $-s$ unterbindet potentielle Resilbifizierungen. Diese strikte Uniformität steht im Dienst der onymischen Schemakonstanz: Das unsilbische, kurze Flexiv schont den Namenkörper und unterstützt ihn damit in seiner Funktion des monoreferenten rigiden Designators (hierzu s. Nübling 2005).

Auf dem Weg zur Uniformierung einstiger Allomorphe zu einem überstabilen Marker spielt auch die Zuweisungsebene eine nicht zu unterschätzende Rolle: Bevor Deflexion - syntagmatisch oder paradigmatisch stattfindet, kündigt sich dieser Prozess in aller Regel durch eine Abwärtsbewegung, eine Abstufung auf der Konditionierungshierarchie für Allomorphe an, wie dies Abb. 1 zeigt.

Im kompliziertesten Fall ist das Allomorph mit seinem Stamm auswendig zu lernen (lexikalisch), d.h. seine Distribution ist idiosynkratisch (s. den Plural bei Atlas - Atlanten). Auch Genus und Flexionsklasse gehören zu den komplexeren Zuweisungsebenen, da diese ihrerseits oft nicht direkt aus dem Lexem erschließbar sind (s. die onymische Flexion im Ahd.). Einfacher wird es bei der semantischen Steuerung, wo z.B. Belebtheit bestimmte Kasus- oder Pluralallomorphe steuern kann (s. etwa die schwachen Maskulina bei den Appellativa). Unterhalb der gestrichelten Linie in Abb. 1 beginnen die formalen Prinzipien, die als einfacher zu gelten haben und die mit morphologisch, prosodisch und phonologisch eine weitere sukzessive Abstufung implizieren. Diachron läutet eine solche Abstufung der Zuweisungsprinzipien von Morphemen in aller Regel eine spätere Deflexion ein: Morphologie vereinfacht sich hier schon in einem ersten Schritt sozusagen hinter den Kulissen. 


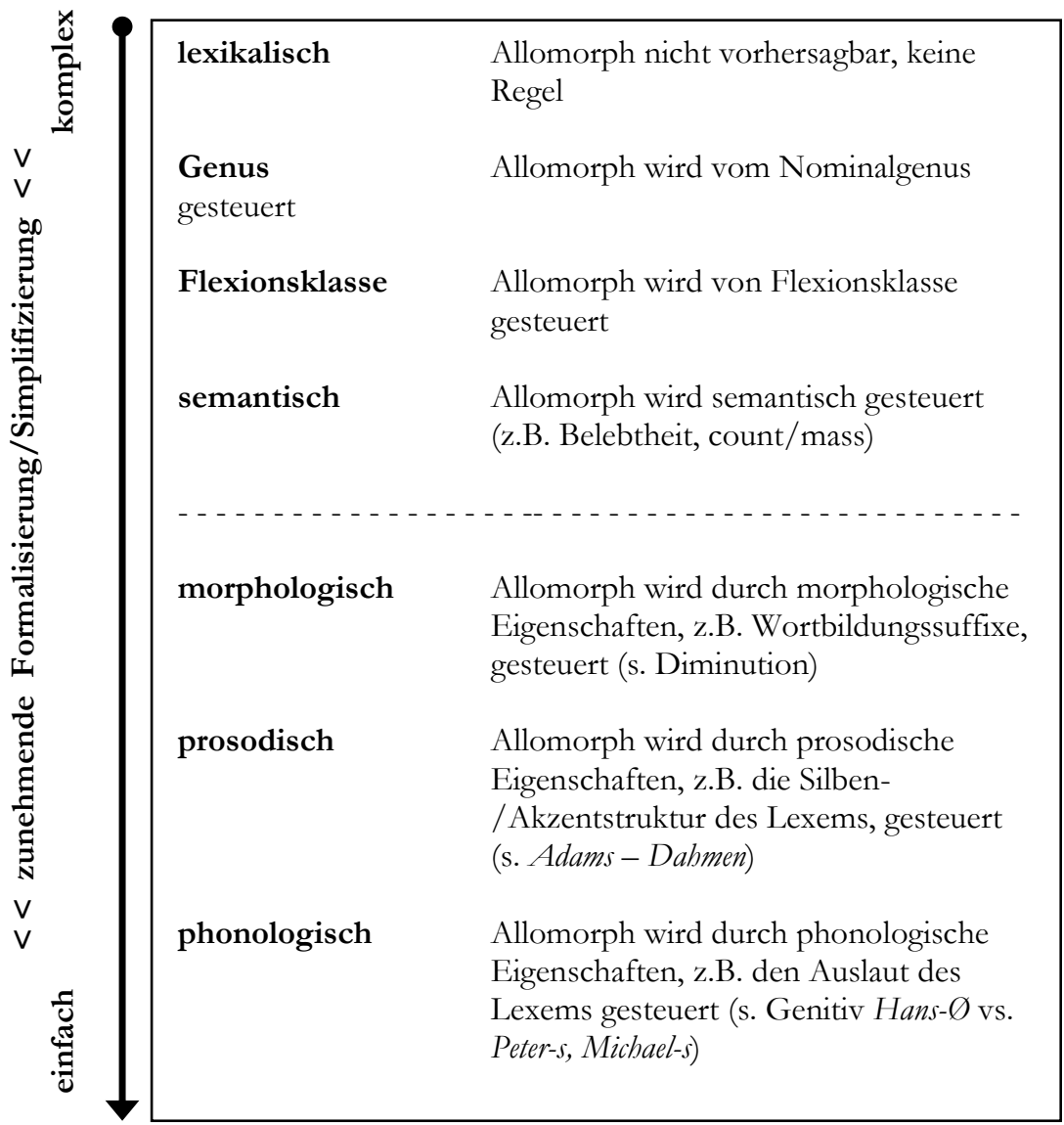

Abb. 1: Hierarchie der Konditionierung von Allomorphen

Auch diese ersten, kaum sichtbaren Schritte der Zuweisungsebenenabstufung lassen sich bei den onymischen Genitivmarkern gut beobachten und kamen bereits zur Sprache: Wenn starke und schwache Klassen kontaminieren und wenn sogar die Genusschranke zwischen Feminina und Maskulina überwunden wird, findet eine dramatische Abstufung statt, die genau dann in eine Formalisierung mündet, wenn materielle Qualitäten des Namenkörpers die Macht über das Allomorph gewinnen. Auf phonologischer Ebene besteht der Fall, dass lange Zeit Namen auf Sibilant das lange Flexiv -ens erhielten (das heute zu Null übergeht), während alle anderen Namenauslaute mit $-s$ suffigiert wurden und werden. Auch für eine prosodische Konditionierung lassen sich Beispiele finden: So nehmen wenn, dann nur Einsilber wie Hans oder Fritz den langen Genitiv (Hansens, 
Fritzens), doch nicht Mehrsilber wie Johannes oder Markus (*Johannesens, *Markusens). Ein anderes Beispiel scheint für das Westmitteldeutsche zu gelten, wo mehrsilbige Rufnamen wie Adam das kurze -s, doch einsilbige das lange -es nehmen, d.h. im Output entstehen immer Trochäen. Dies lässt sich sogar an ein und demselben Namen wie Adam und seiner Kurzform Dahm - beide sind als Familiennamen reich überliefert - dokumentieren: In der Telekom-Datenbank von 2005 gibt es 3.213 Telefonanschlüsse für den starken Genitiv Adams, 2.277 Anschlüsse für den starken Genitiv Dahms, und 2.675 Anschlüsse für schwach gebildetes Dahmen (hinter diesen Zahlen verbergen sich etwa dreimal soviele Namenträger). Nicht ein einziges Mal kommt der schwache Genitiv *Adamen vor. Das heißt, nur in seiner Verkürzung zum Einsilber ist dieser Name in die schwache Flexion übergegangen. Das Verbreitungsareal von schwachem Dabmen ist erstaunlich fest umrissen und konzentriert sich um und vor allem westlich von Köln. Das Adams-Gebiet grenzt direkt südlich daran an (zu formalen Konditionierungen von Rufnamen im Südhessischen s. Mottausch 2004).

Insgesamt hat der onymische $s$-Genitiv eine radikale Generalisierung erfahren, die in der Appellativik nicht ansatzweise so stark ausgeprägt ist, wo sich bis heute viele Flexionsklassen erhalten haben. Dieser onymische Allomorphieabbau geht mit einer Formalisierung und damit Simplifizierung der Zuweisungsebenen einher. Radikal ist dieser Prozess auch in folgender Hinsicht: Während das gesamte Flexionsverhalten der Appellativa diachron auf eine +/- Femininum-Divergenz zusteuert (maskuline und feminine Flexionsklassen werden sich diachron immer unähnlicher), bahnt sich bei den Onymen das genaue Gegenteil einer Maskulinum/Femininum-Konvergenz an. Dabei ist es die maskuline Flexion, die sich durchgesetzt hat. Eigennamen und Appellativa driften somit in ihrem grammatischen Verhalten diachron immer weiter auseinander. Die Eigennamen reduzieren bzw. eliminieren ihre Flexionsallomorphie und betreiben damit auf paradigmatischer Ebene Flexionsabbau: Sie räumen das einst reiche Endungsinventar.

\subsubsection{Syntagmatischer Flexionsabbau}

Die nächste große Entwicklung besteht nun in der syntagmatischen Deflexion: Jetzt schwindet die Flexivik am Namenkörper. Dies betrifft zunächst den Dativ und den Akkusativ bei den Personennamen und bahnt sich ab dem 18. Jh. wie folgt an: 
„Die flektierten Dative und Akkusative auf -(e)n haben allmählich angefangen als vulgär zu gelten. Man hört daher jetzt auch wohl ohne Flexion ich habe Karl (Anna) gebeten, ich habe es Karl (Anna) gegeben. Allgemein ist jetzt die Flexionslosigkeit zur Herrschaft gelangt, wenn daneben ein attributives Adj. oder Pron. steht. Man sagt also Meinem, meinen Karl, meiner, meine Marie. Auch im Gen. pflegt in diesem Falle die Flexion zu unterbleiben, doch ist sie im 18. Jahrh. noch gewöhnlich [...]. Hier ist auch die Verwendung des Art. neben den Eigennamen in Betracht zu ziehen [...].“ (Paul 1917:\$111)

Diese (e)n-Endungen waren auch auf Familiennamen übergetreten (Goethen, Vossen), wo sie ebenfalls abgebaut werden. Eine interessante Begründung für diese Deflexion liefert Steche (1925:205-207, 1927:142), der zu bedenken gibt, dass die Erschließ- und damit Erkennbarkeit des onymischen Stamms (der Nominativform) bei dieser Flexion nicht mehr gegeben war: So lässt sich bei Franken nicht ermitteln, ob der Stamm Frank oder Franke lautet; ebenso könne die Form Heydens auf der Grundform Heyd, Heyde oder Heyden basieren. Dies sei insbesondere auch bei unbekannten Eigennamen, die seit dem 19. Jh. rasant zunehmen, störend.

„Das Störende dieser Doppeldeutigkeit ist tatsächlich in einigen Fällen nachgewiesen; so teilt Wustmann [...] mit, dass zwei Bücher zweier Männer Christ und Weck in Büchereiverzeichnissen meist unter den Namen Christen und Wecken angeführt werden, weil auf den Titeln steht: von Christen, von Wecken." (Steche 1927:142)

Hier zeigt sich deutlich, dass die Flexion die onymische Schemakonstanz beeinträchtigt und dass sie deshalb abgebaut wird (mehr bei Nübling/ Schmuck 2010).

Beim Genitiv stellt sich ab dem 18. Jh. die Tendenz zur sog. Monoflexion ein, d.h. er wird innerhalb der NP nur noch einfach (und nicht mehr kongruierend) realisiert. Befindet sich beim Namen ein Artikel oder Adjektiv, so übernimmt dieser/dieses die Genitivflexion, während der Name zunehmend deflektiert. Steht kein Begleiter, so übernimmt der Name bis heute die Genitivendung, zumindest dann, wenn er ein Possessionsverhältnis ausdrückt wie in dem folgenden Beispiel: der Geburtstag des Peter aber: Peters Geburtstag/der Geburtstag Peters. Diese Varianz gilt auch noch heute, wenngleich der unflektierte Gebrauch des Namens eher die Regel bildet. So schreibt die Duden-Grammatik (2009:\$310): „Maskuline Personennamen mit sekundärem Artikel sind heute überwiegend endungslos, Formen mit der starken Endung $-s$ können aber nicht als falsch bezeichnet werden [...]: die Werke des jungen Dürer, die Kreativität des kleinen Daniel, des alten Wilhelm wohlbekannte Zügec. Daneben werden auch einige Belege mit -s geliefert. Ähnliches schreibt auch Duden-Band 9 „Richtiges und gutes Deutsch“ (2007:698), wohingegen in „Fehlerfreies und gutes Deutsch“" (Wahrig 2003:0S320, 321) die Genitivflexion wie folgt festgelegt wird: 
falsch: der Brief des lieben Georgs

richtig: der Hut des Peter

der Brief des lieben Georg der Hut von Peter

Das heißt, hier entfällt schon bei jeglichem Erscheinen eines Substantivbegleiters -s am Personennamen. Außerdem wird der von-Anschluss statt des nachgestellten Genitivs empfohlen, der - neben der damit bewirkten Deflexion - auch das Possessionsverhältnis besser ausdrücken könnte. Deutlich wird, dass es sich hier um einen echten gegenwärtigen Zweifelsfall handelt. Mit dieser Tendenz zur Monoflexion erweisen sich NPs mit Eigennamen als progressiv im Gegensatz zur sonst weithin geltenden Wortgruppenflexion (Duden-Grammatik 2009:\$1517). Bei den femininen Personennamen steht strikt nur dann Genitiv-s, wenn kein weiterer Begleiter vorhanden ist: Annas Geburtstag - der Geburtstag Annas, aber: der Geburtstag der großen Anna (zu dem Zusammenhang zwischen Artikelsetzung und Feminina insbesondere bei Territorialnamen s. Thieroff 2000).

Etwas anders sieht es bei Toponymen aus, die der Entwicklung der Personennamen hinterherzuhinken scheinen. Hierzu schreibt die DudenGrammatik (2009:\$309):

„Endungslose Formen werden nicht mehr als falsch angesehen, vor allem bei fremdsprachlichen Namen. Mehrsilbige Namen, die auf eine unbetonte Silbe mit $s$-Laut ausgehen, haben in der Regel endungslose Formen im Genitiv:

der Neckar $\rightarrow$ des Neckars (seltener: des Neckar); der Tiber $\rightarrow$ des Tibers (seltener: des Tiber), der Mississippi $\rightarrow$ des Mississippi (oder: des Mississippis); der Ob $\rightarrow$ des Ob (oder: des Obs), der Amazonas $\rightarrow$ des Amazonas (nur so); der Ganges $\rightarrow$ des Ganges (nur so)"

Auch Wahrig (2003:§323) „Fehlerfreies und gutes Deutsch“ lässt hier noch in vielen Fällen das Genitiv-s gelten:

„Die mit Artikel gebrauchten (maskulinen und neutralen) geographischen Eigennamen stehen zunehmend ohne Genitiv-s. Das Genitiv-s steht allerdings noch häufig bei Namen deutscher Flüsse: des Rheins, Mains, Neckars (auch: des Neckar), Inns (auch: des Inn).

Bei den meisten mit Artikel gebrauchten geographischen Eigennamen überwiegt jedoch die Form ohne -s [...]: des Atlantik(s), des Ätna(s), des Balkan(s), des Engadin(s), des Himalaja(s), des Iran(s), des Mississippi(s), des Kongo(s), des Spessart(s)“.

Geographische Namen mit Attribut stünden „heute vorwiegend ohne $-s$, doch ist auch die Form mit $-s$ immer möglich: die Skyline des modernen Frankfurt(s), die Mitte des neuen Berlin(s), des wiedervereinigten Deutschland(s) ...." (ebd.). ${ }^{11}$

11 Besondere Aktualität hat die Flexion weiterer komplexer Eigennamentypen, etwa von Titeln von Büchern, Theaterstücken, Opern, Zeitschriften etc. Vgl. die häufig gestellten An- 
Dagegen schreibt gut 100 Jahre früher Blatz (1900:346/347), dass bei einem Attribut vor geographischen Namen noch Doppelflexion erfolge: die Ufer des lieblichen Rheins; die Wälder des rauhen Spessarts, die Völker des alten Galliens. Doch werde ,in neuerer Zeit" die onymische Flexion unterlassen: die Völker des heißen Afrika, die Ufer des lieblichen Neckar. Doppelflexion gelte indessen nicht mehr für Personennamen: die Kriege des tapfern Caesar, des grausamen Nero. Allerdings handle es sich hier um neuesten Sprachgebrauch, denn bis zur Mitte des 19. Jhs. sei es „nicht unüblich“ gewesen, noch doppelt zu flektieren: die Worte des (erzürnten) Luthers, das Fest des (heiligen) Josephs, unseres (vortrefflichen) Lessings.

Dieser Rundgang macht deutlich: Bis ca. 1850 scheint die Polyflexion in NPs mit Eigennamen noch üblich gewesen zu sein. Dann erfolgt der onymische Flexionsverlust hin zur Monoflexion, zunächst bei Personennamen, gegen 1900 auch bei geographischen Namen. Dabei scheint die Belebtheitshierarchie zu wirken. Außerdem wird deutlich, dass die Bekanntheit eines Namens bzw. generell native Strukturen eher das Genitiv-s erlauben als seltene, fremde Namen (Eisenberg 2006, II:164).12 Dies weist deutlich darauf hin, dass die Deflexion im Dienst der Schonung des Wortkörpers und dessen besserer Wiedererkennbarkeit steht.

Mit zunehmender Deflexion etabliert sich derzeit immer mehr, von Süden nach Norden ausstrahlend, die Verwendung des Definit- bzw. Expletivartikels bei Personennamen: „Entsteht Undeutlichkeit des Kasus, so tritt der Artikel ein, z.B. Ich ziehe Schiller dem Göthe vor. Karl besucht den Adolf* (Blatz 1900:336; zur Diskussion des Status von Artikeln bei Eigennamen s. Gallmann 1997, zum Artikelgebrauch heute Bellmann 1990). Dieser Artikel ist in Süddeutschland durchgrammatikalisiert (expletiv) und dürfte primär die fehlende Kasusmarkierung kompensieren. ${ }^{13}$ Wie der folgende Satz zeigt, ergeben sich a) mit bzw. b) ohne Artikel vollkommen unterschiedliche Lesarten, d.h. im Fall von b) Artikellosigkeit wird die Satzgliedstellung funktionalisiert:

fragen bei Sprachberatungsstellen, bspw. ob es heiße, Gestern waren wir im Fliegenden Holländer oder Gestern waren wir in "Der Fliegende Holländer"

12 Ganz explizit macht dies Steche (1927:148) zur Regel: „Deutsche Nichtmenschennamen erhalten die Endung $-s$, fremde sind endungslos"

13 Anders verhält es sich im weitgehend artikelfreien Norden und in einem Übergangsstreifen Duisburg, Kassel, Erfurt, Leipzig, Dresden: Am ehesten wird hier der Artikel bei negativer Expressivität und im Obliquus gesetzt, am wenigsten bei der Selbstreferenz oder der Referenz auf im Raum anwesende oder auch nur sichtbare (Fotografie) Personen (hierzu s. eingehend Bellmann 1990: 257-282). Insgesamt sieht auch Bellmann einen deutlichen Zusammenhang zwischen der Namendeflexion und der kompensierenden Artikelverwendung. Teilweise ist die norddeutsche Artikellosigkeit noch im Niederdeutschen vorhandenen Obliquusflexiven geschuldet: In Magdeburg, Halle und Leipzig verwenden die Älteren noch die Form Petern: Gib das Petern. Kennst du Petern? (Bellmann 1990:276) 
a) Dem Peter[Dat.] hat derDaniel[Nom.] den Stefan[Akk.] empfoblen b) $\neq \operatorname{Peter}[$ Nom. $] \quad$ hat Daniel[Dat.] Stefan[Ak.k.] empfoblen

Im gegenwärtigen Standarddeutschen ist eine weitere Stufe zur Deflexion $\mathrm{zu}$ verzeichnen: Das bis dato phonologisch konditionierte Genitivallomorph -ens nach Sibilant schwindet zunehmend: ?Hans-ens > Hans' Geburtstag, Marxens > Marx” „Kapital“. Auch hierzu sei die Duden-Grammatik (2009:\$323) konsultiert: „Bei Eigennamen, die im Nominativ auf einen $s$-Laut ausgehen [...], verschmilzt die Genitivendung damit. In geschriebener Sprache wird die Verschmelzung mit dem Apostroph angezeigt. Solche Formen erscheinen fast nur noch als vorangestellte Genitivattribute: Fritz' Hut [...], Onassis' Jachten“. Duden Band 9 (,Zweifelsfälle“) bezeichnet die lange ens-Endung als ,altertümlich“ und empfiehlt als Alternative zum blanken, flexionslosen Namen die von-Umschreibung oder die Artikelverwendung: der Geburtstag von Hans / des Hans. Wie Steche (1927:147) hervorhebt, erfahren besonders sog. „Nichtmenschennamen“ im Genitiv die von-Umschreibung (der Bürgermeister von Bremen, das Zentrum von Nürnberg). In jedem Fall ist hier die komplette onymische Deflexion erreicht. Die vonUmschreibung dürfte diachron den nächsten (und letzten) Schritt darstellen.

Abschließend bleibt noch festzustellen, dass mit diesen formalen auch funktionale Reduktionen des onymischen $s$-Genitivs einherzugehen scheinen, indem er sich, ähnlich wie im Englischen, zu einem Possessiv entwickelt: Annas Hund, Peters Katze (hierzu s. eingehend Eisenberg/Smith 2002). Wird der Genitiv dagegen von einer Präposition regiert, bleibt der Name eher unflektiert: sie kam trotz des Wetters vs. sie kam ?trotz Peters, ?trotz. Annas. Sobald sich Peters oder Annas jedoch auf ein Bezugsnomen beziehen (und sich damit der Rektion von trotz entziehen), kann diese Konstruktion wie folgt fortgesetzt werden: sie kam trotz. Annas (Peters) Hund. Diese besondere Unterdrückung des „echten“ Genitiv-s beobachtet schon Blatz (1900): „In Abhängigkeit von einigen Präpositionen (Adverbien) des Orts, die den Genet. regieren, wie oberhalb, unterhalb, diesseit(s), jenseit(s), außerhalb, innerhalb, unweit entbehren Städtenamen häufig das Genetivzeichen -s, z.B. Innerhalb Berlin, unweit Hamburg. Jenseits Salzburg“" (341).

Fazit: Alles in allem zeichnet sich der Eigenname im Vergleich zu seinem nächsten Verwandten und diachronen Vorgänger, dem Appellativ, durch Flexionsarmut bis hin zu Flexionslosigkeit aus: Heute besteht nur noch ein onymisches Einklassensystem (fast) ohne genussensitive morphologische Unterschiede sowie (fast) ohne jegliche Allomorphie (paradigmatische Deflexion) bei extrem "sparsamer" (Mono-)Flexion (syntag- 
matische Deflexion). Diese Restflexion wird zunehmend durch die vonUmschreibung abgelöst und damit obsolet. Funktional verengt sich der Genitiv zum Possessiv.

\section{$2.3 \quad$ Numerus}

Eine sehr viel kleinere Rolle als die Kasusflexion spielt die Pluralflexion bei Eigennamen, da Eigennamen per definitionem monoreferente Einheiten sind. Der Plural bei Eigennamen bezeichnet namensgleiche Träger und ist als grammatische Form spät bezeugt. Erst im Frühneuhochdeutschen kommen vermehrt Namenplurale auf (Paul 1917:\$113).

Auch bzgl. der Pluralbildung weisen die Eigennamen zunächst fast die gleichen Allomorphe wie die Appellativa auf, auch durchaus solche mit Umlaut, was heute nicht mehr möglich ist (ebd.). Dabei lassen die Maskulina die starke Flexion (Plural-e) erkennen, die Feminina dagegen sowohl eine starke (auf -s) als auch eine schwache (auf -(e)n). Dabei gilt gemäß Paul (1917:158), dass die Feminina mit $s$-Genitiv auch ihren Plural mit $-s$ bilden, die mit einem ens-Genitiv dagegen mit -en:

Maskulina: die beiden Hänse (vgl. noch heute die appellativisch verwendeten Prablhänse), Kläuse, Heinriche, Ottone, Stolberge, Lessinge, Klopstocke, Sokratesse;

Feminina: $\quad$ stark: die beiden Annas, Evas, Kathis; schwach: die beiden Susannen, Luisen, Marien, Helenen.

Diese Parallelität zwischen $s$-Genitiv und $s$-Plural deutet darauf hin, dass das onymische Plural-s seine Herkunft aus einer Reanalyse der Genitivform bezieht: Wie in Nübling/Schmuck (2010) ausführlich dargelegt wird, hat diese Reanalyse bei Familiennamen über Brückenkonstruktionen wie die folgende stattgefunden: Wir sind bei des Müllers (,Gen.Sg.') Familie/ Leuten $>$ Wir sind bei des Müllers [Familie/Leuten: wird elliptisch ausgelassen] $>$ wir sind bei ('s) Müllers, $\mathrm{Pl} .{ }^{\prime}$ - Müllers (,Pl.') haben (,Pl.') sich wieder gemeldet. Deutlichste Evidenz dafür, dass es tatsächlich solche elliptischen Konstruktionen waren, liefern süddt. Dialekte, die den Gen.Sg.-Artikel noch proklitisch mit dem mittlerweile pluralischen Namen atavistisch fortsetzen: mir sin bi's Müllers („Wir sind bei des Müllers“ $\rightarrow$,Wir sind bei Müllers'); s'Müllers sin do („Des Müllers sind da“ $\rightarrow$,Müllers sind da). Dieses Plural-s hat langfristig, ausgehend von den Familiennamen und von dort übergreifend auf die Ruf- und später die Ortsnamen, sämtliche Namenklassen erfasst.

Für eine weitere augenscheinliche Parallele zwischen Genitiv und Plural spricht die Formgleichheit der Doppelendung -ens bei Familiennamen, die auf [s] auslauten: die beiden Marx-ens, Kunz-ens, Jens-ens. 
Hierzu schreibt Steche (1927:149): „Die lautlichen Verhältnisse [der Pluralbildung] sind ebenso wie bei der Unterfallendung [Genitivendung] s: Schmidts, Grotes [...]. Nach s-Lauten verwende man die Endung -es oder -ens: Fuchses, Hinrichses besser als Fuchsens, Hinrichsens, Kunzes zu dem Namen Kunze und Kunzens zu Kunz". Heute ist der ens-Plural zwar nicht mehr sehr geläufig (er wird mit -es oder nullrealisiert), doch durchaus noch bekannt.

Bemerkenswert ist die Tatsache, dass es früher, gerade bei den Maskulina, viele Pluralallomorphe gab, sogar solche, die den Namenkörper durch (morphologischen) Umlaut affizierten: Paul (1917:159) schreibt, dass Hans anfänglich schwach pluralisiert wurde (Hansen), später jedoch stark (Hänse). Im Laufe des 19. Jhs. schwinden jedoch die meisten Allomorphe, nämlich $-e, \mathrm{UL}+-e$ und $-e n$. Die Nullendung verbindet sich noch länger mit auf -er auslautenden Namen (wie Peter, Dieter), doch auch hier ist das Plural-s die mittlerweile üblichere Endung (in meiner Klasse gibt es zwei Peters). Damit hat auch beim onymischen Plural eine radikale Uniformierung stattgefunden, und auch hier ist es - sicher nicht zufällig - das -s, das sich durchgesetzt hat. Indem der Umlaut aus dem Namenkörper beseitigt wird, wird dieser konstant gehalten. Das gleiche ist auch beim Diminutivumlaut eingetreten, der heute nur noch bei einer kleinen Restschicht alter Namen fortlebt: Hänschen klein, Hänsel, Kläuschen. Heute ist er bei Eigennamen längst nicht mehr produktiv (im Gegensatz zu Appellativa): Hanne Hannchen, Susanne - Susannchen, Jan - Janchen, Paul - Paulchen. Auch hier wird sichtbar, wie der Namenkörper nach und nach durch rein suffigierende Flexion oder gar durch Nullflexion möglichst unangetastet bleibt.

Heute wird dieser wortschonende $s$-Plural jedoch zunehmend durch ein noch schonenderes Verfahren ersetzt, den Nullplural: Ähnlich dem Genitivausdruck schwindet auch der Pluralausdruck dann am Namenkörper, wenn er an einem Begleitwort, meist dem Artikel, zum Ausdruck kommt. Auch hier entsteht damit Monoflexion: die beiden Peter(s), die beiden Deutschland(s). In welchem Ausmaß solche gegenwärtigen Schwankungen bestehen, darüber soll das nächste Kapitel Auskunft geben.

\subsection{Korpusbefunde zum onymischen Genitiv- und Pluralausdruck}

Um die genauen Schwankungen besser greifen zu können, wurden im April 2010 Korpusuntersuchungen in Cosmas angestellt. Zunächst wird die Genitivmarkierung bei solchen Gebirgs-, Landschafts- und Ländernamen untersucht, die einen festen Artikel und damit einen Genitivträger bei sich führen (Tab. 3). 


\begin{tabular}{|l|l|l|l|}
\hline Name im Genitiv & \multicolumn{2}{|l|}{ Null-Endung } & s-Endung \\
\hline & Prozent & abs. & abs. \\
\hline des Himalay Jja- & $85 \%$ & 775 & 132 \\
\hline des Jemen- & $83 \%$ & 217 & 43 \\
\hline des Kongo- & $73 \%$ & 495 & 185 \\
\hline des Irak- & $68 \%$ & 3894 & 1856 \\
\hline des Iran- & $65 \%$ & 1916 & 1024 \\
\hline des Europa- & $46 \%$ & 36 & 43 \\
\hline des Balkan- & $23 \%$ & 317 & 1075 \\
\hline des Engadin- & $7,6 \%$ & 19 & 229 \\
\hline des (wieder)verein(ig)ten Deutschland- & $52 \%$ & 224 & 205 \\
\hline
\end{tabular}

Tab. 3: Der Genitiv bei Gebirgs-, Landschafts- und Ländernamen mit Artikel (CosmasRecherche IDS); graue Hinterlegung: dominante Flexion

Deutlich wird durch die grauen Hinterlegungen, dass die Nullendung insgesamt stärker repräsentiert ist. Die Bekanntheit bzw. Nativität des $\mathrm{Na}$ mens scheint dabei eine Rolle zu spielen und das Genitiv-s eher zu fördern: Europa, Balkan und Engadin haben hier höhere Werte als beim NullGenitiv; bei Deutschland in der letzten Zeile hält es sich ungefähr die Waage. Es liegt auf der Hand, dass solche Untersuchungen noch auf eine größere Basis gestellt werden müssen. Das Prinzip, dass bekannte (= häufig versprachlichte) Namen eher durch ein Flexiv verfremdet werden dürfen als exotische, bestätigt sich auch bei den Flussnamen (Tab. 4): Rhein, Neckar und Nil führen das Genitiv-s im Gegensatz zu den fremderen Flussnamen. Da die Flussnamen geringere Zahlen erbracht haben, wurden hier keine Prozentwerte errechnet. Steche (1925:205 ff.) geht sogar so weit zu sagen, dass es gerade die seit dem 19. Jh. durch Handel und Bildung stark zunehmenden fremden Eigennamen sind, die den Anstoß zur onymischen Deflexion gegeben haben, um ihren (weitgehend unbekannten) Wortkörper erschließ- und erkennbar zu halten. ${ }^{14}$

14 Steche (1925:206): „Im Jahre 1800 wird [....] der gebildetste Deutsche in seinem ganzen Leben nicht soviel fremde Eigennamen gekannt haben, wie heute in einer einzigen Nummer einer großen Zeitung stehen (man denke allein an den Handelsteil)“. 


\begin{tabular}{|l|l|l|}
\hline Name im Genitiv & Null-Endung & s-Endung \\
\hline des Rhein- & 64 & 11.393 \\
\hline des Neckar- & 44 & 935 \\
\hline des Nil- & 94 & 457 \\
\hline des Mississippi- & 365 & 22 \\
\hline des Orinok|co- & 62 & 2 \\
\hline des Tiber- & 48 & 30 \\
\hline des Yangtse- & 59 & 0 \\
\hline
\end{tabular}

Tab. 4: Der Genitiv bei Flussnamen (Cosmas-Recherche IDS)

Dass es primär der Eigennamenstatus ist, der flexionsunterdrückend wirkt, und nicht sein Fremdheitsgrad, zeigt abschließend das Praxonym der 11. September, das euphemisierend den größten Terrorangriff auf die USA bezeichnet. Dieser Name wird in Tab. 5 mit formal ähnlich strukturierten Zeitangaben kontrastiert.

\begin{tabular}{|l|l|l|l|}
\hline & $\begin{array}{l}\text { Bezeichnung im } \\
\text { Genitiv }\end{array}$ & Null-Endung & s-Endung \\
\hline Name & des 11. September- & $2079=86 \%$ & $347=14 \%$ \\
\hline \multirow{2}{*}{ Zeitangaben } & des 19. Jabrbundert- & $332=1,5 \%$ & $21.600=98,5 \%$ \\
\cline { 2 - 4 } & des 20. Jabrbundert- & $251=1 \%$ & $22.266=99 \%$ \\
\hline
\end{tabular}

Tab. 5: Das Praxonym der 11. September im Vergleich mit anderen Zeitbezeichnungen (CosmasRecherche IDS)

Hier zeigt sich deutlich, dass beim Praxonym die Unterdrückung des Genitiv-s stark überwiegt, während die Zeitangaben es fast immer mit sich führen.

Was die Pluralflexion betrifft, so liefert Cosmas zu wenig Belege, als dass sich valide Aussagen treffen ließen. Um etwas mehr Belege zu erzielen, wurden alle Kasusformen abgefragt. Es ergibt sich ungefähr ein hälftiges Bild, d.h. ein echter Zweifelsfall (Tab. 6).

\begin{tabular}{|l|l|l|}
\hline Name im Plural & Null-Endung & s-Endung \\
\hline die beiden Deutschland- & 11 & 6 \\
\hline der beiden Deutschland- & 8 & 10 \\
\hline den beiden Deutschland- & 14 & 14 \\
\hline $\begin{array}{l}\text { Summe: } \\
\text { absolut }\end{array}$ & 33 & 30 \\
\hline Prozent & $53 \%$ & $47 \%$ \\
\hline
\end{tabular}

Tab. 6: Die Pluralmarkierung bei die beiden Deutschland(s) (Cosmas-Recherche IDS) 


\section{Die Deflexion im Überblick}

Zusammenfassend lassen sich die Etappen der onymischen Deflexion mit dem Schaubild in Abb. 2 illustrieren.

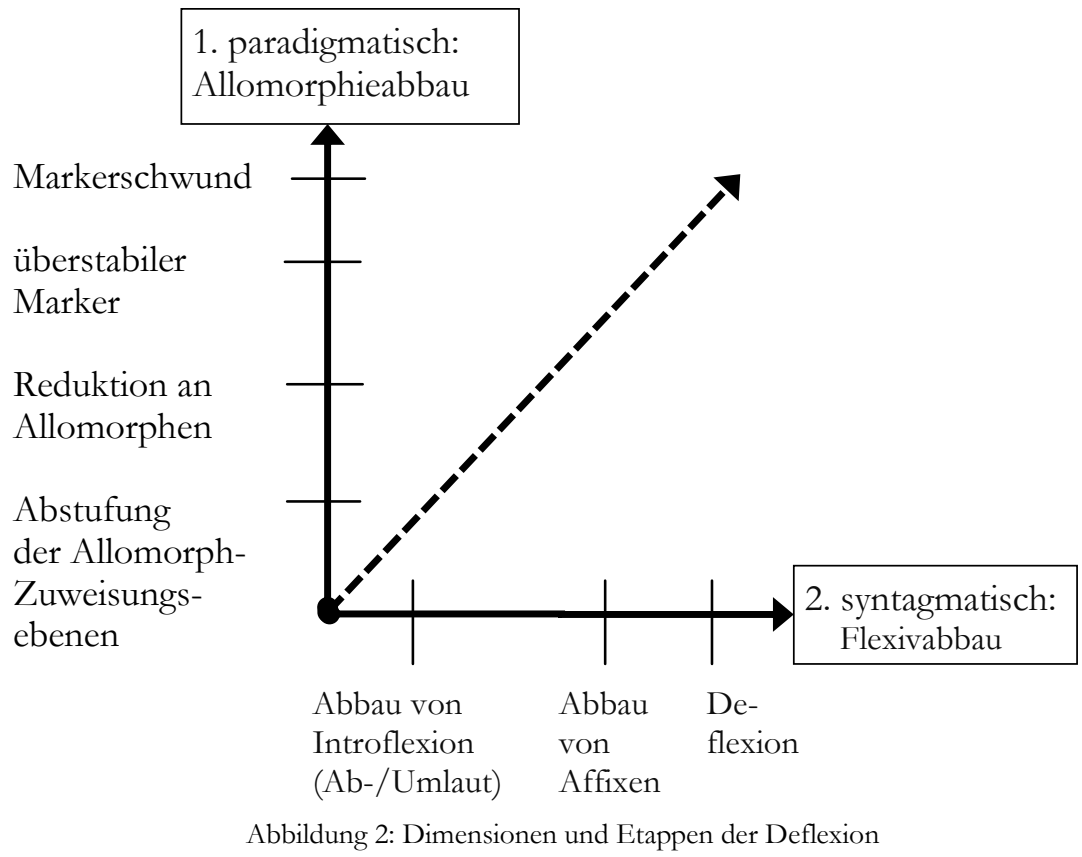

Prinzipiell beginnt Deflexion auf der paradigmatischen Ebene, zunächst auf wenig sichtbare Weise, indem bei der Steuerung der Allomorphe ein Komplexitätsabbau bzgl. der Zuweisungsebenen stattfindet: Die Allomorphe entkoppeln sich von ihren Flexionsklassen, ja sogar vom Genus ihres Nomens, und folgen sukzessive formaleren Steuerungsprinzipien: Silbenzahl, Wortumfang, Wortauslaut etc. bestimmen zunehmend ihre Distribution. Im Gefolge dieses Simplifizierungsprozesses reduziert sich auch die Zahl der Allomorphe. Es bilden sich schließlich überstabile Marker heraus, die im Gegensatz zu natürlichkeitstheoretischen Annahmen gerade nicht von starker, sondern von geschwächter Morphologie zeugen: Allomorphie ist ein Ausweis starker und Markerüberstabilität von sich abbauender Morphologie (hierzu Dammel/Nübling 2006). Diesen Zusammenhang bestätigt die Geschichte der onymischen Morphologie, denn während des paradigmatischen Abbaus haben sich auch auf der syntagmatischen Achse Deflexionserscheinungen eingestellt. 
Was den Namenkörper selbst betrifft, so entledigt er sich nach und nach seiner Flexivik: Zunächst ziehen sich die ihn besonders stark modifizierenden introflektierenden Verfahren (wie der Umlaut) zurück, der Wortkörper gewinnt an Autonomie/Stabilität. In einem weiteren Schritt befreit sich der Name auch von den Affixen an seiner Peripherie, wobei er den Ausdruck der Nominalkategorien auf seine Begleiter verlagert. Dass dieser gesamte Prozess funktional mit der Monoreferenz zu begründen ist, wurde mehrfach herausgestellt: Namen referieren direkt auf ein Objekt und sollten maximal konstant sein, zumal sie oft nichtnative Strukturen enthalten. Wahrscheinlich bilden sie diejenige Kategorie mit den wenigsten nativen Elementen. Umso mehr bedürfen sie der Strukturschonung.

Während sich die paradigmatische Dimension der Deflexion schon im Mhd. einstellt, erfolgt die syntagmatische Deflexion erst ab dem 18. Jh. und schreitet bis heute unvermindert fort, erkennbar an der Vielzahl von Zweifelsfällen. Am deutlichsten formuliert dies Steche (1927:140), wenn er schreibt:

„Die Eigennamen sind die einzigen Wörter der deutschen Sprache, die im 19. Jahrhundert eine tiefgreifende Umgestaltung erfahren haben; die übrige Sprache stimmt dagegen noch fast völlig mit derjenigen unserer großen klassischen Dichter überein. Diese Unterschiede bei den Eigennamen sind altbekannt, aber eine Erklärung findet man eigentlich nie [...]. In der Beugung hat sich während des 19. Jahrhunderts der Verlust der Endungen durchgesetzt.“

Keine andere Einheit ist in der jüngsten Sprachgeschichte von solch tiefgreifenden Umstrukturierungen affiziert worden wie der Eigenname. Dazu zählt auch sein Genusverhalten bzw. seine Genuszuweisung: Der Eigenname entwickelt sich immer mehr zu einer Einheit mit einem feldextern vergebenen, pragmatischen-referentiellen Genus. Dass wir so wenig Genaues über seine Diachronie wissen, liegt daran, dass in den Darstellungen zur historischen Nominalflexion die Eigennamen bestenfalls in Fußnoten abgedrängt, in aller Regel jedoch gar nicht berücksichtigt werden. ${ }^{15}$ Dies hat uns bisher die Sicht darauf verstellt, dass sich die Eigennamen flexionsmorphologisch mehr und mehr von den Appellativen abgespalten und in Richtung einer eigenen Wortart entwickelt haben. Es ist tatsächlich die Frage zu stellen, was noch geschehen müsste, um eine Wortart „Eigenname" zu legitimieren. Dass sie sich auch syntaktisch immer mehr von den anderen Substantiven distanzieren, ist diachron ebenfalls nachweisbar.

15 Eine Ausnahme davon wird die derzeit entstehende, korpusbasierte Mittelhochdeutsche Grammatik bilden, deren Teil zur Substantivflexion von Klaus-Peter Wegera erarbeitet wird. Für Einblicke vorab in das Kapitel „Flexion der Personennamen“ sei ihm an dieser Stelle sehr gedankt. 


\section{Literatur}

Bellmann, Günter (1990): Pronomen und Korrektur. Zur Pragmalinguistik der persönlichen Referenzformen. Berlin/New York: de Gruyter.

Blatz, Friedrich (19003): Neuhochdeutsche Grammatik mit Berücksichtigung der historischen Entwicklung der deutschen Sprache. Karlsruhe: Lang.

Dammel, Antje \& Nübling, Damaris (2006): The Superstable Marker as an Indicator of Categorial Weakness?. In: Folia Linguistica XL/1-2, 97-113.

Duden Band 4 (2009): Grammatik der deutschen Gegenwartssprache. 8. Aufl. Mannheim: Dudenverlag.

Duden Band 9 (2007): Richtiges und gutes Deutsch. Wörterbuch der sprachlichen Zweifelsfälle. Mannheim: Dudenverlag.

Eisenberg, Peter (2006): Grundriss der deutschen Grammatik. Band 2: Der Satz. Stuttgart: Metzler.

Eisenberg, Peter \& Smith, George (2002): Der einfache Genitiv. Eigennamen als Attribute. In: Peschel, Corinna (Hrsg.): Grammatik und Grammatikvermittlung. Frankfurt: Peter Lang, 113-126.

Fahlbusch, Fabian (2009): Von KarstadtQuelle zu Arcandor, von MTU Friedrichshafen zu Tognum. Prinzipien des diachronen Wandels von Unternehmensnamen. Magisterarbeit. Universität Mainz.

Fleischer, Wolfgang (1970): Onomastische Strukturen in der deutschen Sprache der Gegenwart. In: OSGV 62, Heft 2, 35-44.

Gallmann, Peter (1997): Zur Morphosyntax der Eigennamen im Deutschen. In: Löbel, Elisabeth \& Rauh, Gisa (Hrsg.): Lexikalische Kategorien und Merkmale. Tübingen: Niemeyer, 72-84.

Köpcke, Klaus-Michael \& Zubin, David (1983): Die kognitive Organisation der Genuszuweisung zu den einsilbigen Nomen der deutschen Gegenwartssprache. In: Zeitschrift für Germanistische Linguistik 11/2, 166-182.

Köpcke, Klaus-Michael \& Zubin, David (1984): Sechs Prinzipien für die Genuszuweisung im Deutschen: Ein Beitrag zur natürlichen Klassifikation. In: Linguistische Berichte 93, 26-50.

Köpcke, Klaus-Michael \& Zubin, David (1996): Prinzipien für die Genuszuweisung im Deutschen. In: Lang, Ewald \& Zifonun, Gisela (Hrsg.): Deutsch typologisch. IdS Jahrbuch 1995. Berlin/New York: de Gruyter, 473-491.

Köpcke, Klaus-Michael \& Zubin, David (2005): Nominalphrasen ohne lexikalischen Kopf - Zur Bedeutung des Genus für die Organisation des mentalen Lexikons am Beispiel der Autobezeichnungen im Deutschen. In: Zeitschrift für Sprachwissenschaft 24, 93-122.

Köpcke, Klaus-Michael \& Zubin, David (2009): Genus. In: Hentschel, Elke \& Vogel, Petra (Hrsg.): Deutsche Morphologie. Berlin: de Gruyter, 132-154.

Kunze, Konrad (20034): dtv-Atlas Namenkunde. Vor- und Familiennamen im deutschen Sprachgebiet. München: Deutscher Taschenbuch Verlag.

Mottausch, Karl-Heinz (2004): Familiennamen als Derivationsbasis im Südhessischen: Bezeichnungen von Familien und Frauen in der Synchronie und Diachronie. In: Zeitschrift für Dialektologie und Linguistik 71, 307-330.

Nübling, Damaris (2005): Zwischen Syntagmatik und Paradigmatik: Grammatische Eigennamenmarker und ihre Typologie. In: Zeitschrift für Germanistische Linguistik $33,25-56$. 
Nübling, Damaris (2009): Von Monika zu Mia, von Norbert zu Noab: Zur Androgynisierung der Rufnamen seit 1945 aus prosodisch-phonologischer Perspektive. In: Beiträge zur Namenforschung 1, 67-110.

Nübling, Damaris \& Schmuck, Mirjam (2010): Die Entstehung des s-Plurals bei Eigennamen als Reanalyse vom Kasus- zum Numerusmarker. Evidenzen aus der deutschen und niederländischen Dialektologie. In: Zeitschrift für Dialektologie und Linguistik 77, Heft 2, 145-182.

Oelkers, Susanne (2003): Naming Gender. Empirische Untersuchungen zur phonologischen Struktur von Vornamen im Deutschen. Frankfurt: Peter Lang Verlag.

Paul, Hermann (1917): Deutsche Grammatik, Band II, Teil III: Flexionslehre. Tübingen: Niemeyer. Nachdruck von 1968.

Paul, Hermann (2007): Mittelhochdeutsche Grammatik. Tübingen: Niemeyer.

Schatz, Josef (1927): Althochdeutsche Grammatik. Göttingen: Vandenhoeck \& Ruprecht.

Seibicke, Wilfried (20082): Die Personennamen im Deutschen. Berlin/New York: de Gruyter.

Steche, Theodor (1925): Neue Wege zum reinen Deutsch. Breslau: Hirt.

Steche, Theodor (1927): Die neuhochdeutsche Wortbiegung unter besonderer Berücksichtigung der Sprachentwicklung im 19. Jahrhundert. Breslau: Hirt.

Szczepaniak, Renata (2010): Während des Flug(e)s/des Ausflug(e)s? German Short and Long Genitive Endings between Norm and Variation. In: Lenz, Alexandra \& Plewnia, Alexander (Hrsg.): Grammar Between Norm and Variation. Frankfurt: Peter Lang Verlag, 103-126.

Thieroff, Rolf (2000): *Kein Konflikt um Krim. Zu Genus und Artikelgebrauch von Ländernamen. In: Hess-Lüttich, Ernest \& Schmitz, Walter (Hrsg.): Botschaften verstehen. Kommunikationstheorie und Zeichenpraxis. Frankfurt: Peter Lang, 271-284.

Wahrig Band 5 (2003): Fehlerfreies und gutes Deutsch. Gütersloh: Bertelsmann. 